\title{
EFECTO DE LA DESHIDRATACION EN LA AGUDEZA VISUAL
}

\author{
Alfredo López Dávila y Walter Salazar Rojas \\ Escuela de Educación Física y Deportes \\ Universidad de Costa Rica, San José, Costa Rica \\ E-mail: wsalazar@cariari.ucr.ac.cr
}

\begin{abstract}
Resumen
López Dávila, A. y Salazar Rojas, W. (2002). Efecto de la deshidratación en la agudeza visual. Revista de Ciencias del Ejercicio y la Salud, 2(2), 11-22. El objetivo de este estudio fue determinar si existe un efecto negativo agudo de la deshidratación inducida por ejercicio sobre la agudeza visual. La evidencia previamente revisada señala que las funciones visuales son de especial importancia en la vida cotidiana, así como en el contexto competitivo. La deshidratación afecta negativamente algunas tareas motrices, disminuye sensiblemente el rendimiento deportivo y genera alteraciones fisiológicas importantes. Por su parte, la sola práctica de la actividad física (sin deshidratación de por medio) genera alteraciones en algunas funciones visuales, tales como la agudeza visual, la cual se ve disminuida, y la visión periférica, que se aumenta. Estos dos efectos son de carácter agudo. A partir de la evidencia existente se decidió evaluar si la deshidratación (2\% de la masa corporal) entre sus efectos fisiológicos afecta negativamente la agudeza visual, variable vital en el contexto competitivo. No se encontró un efecto significativo de la deshidratación sobre la agudeza en el presente estudio. Únicamente se encontraron tales diferencias entre distintos días de aplicación de las pruebas de la agudeza visual. Las variaciones se dieron al evaluar los dos ojos simultáneamente $(p=0.12)$ al evaluar al ojo derecho por sí solo $(p=0.30)$ y no al evaluar al ojo izquierdo por sí solo $(p=0.79)$. Los resultados indican que la agudeza visual se altera significativamente de un día a otro, pero que esto es independiente de la deshidratación, la cual no afecta a la variable dependiente de este estudio. PALABRAS CLAVES: Agudeza visual, visión periférica, deshidratación.
\end{abstract}

\section{INTRODUCCIÓN}

Se considera que la visión borrosa produce un mayor impacto funcional en el individuo, además de afectar más severamente su calidad de vida que padecimientos tales como la hipertensión, la diabetes mellitus II, el infarto al miocardio, la indigestión, los problemas urinarios y la jaqueca (Lee, Spritzer y Hays, 2000).

En el contexto de la actividad física, la información sensorial que el deportista recibe es derivada esencialmente de su sistema visual. El rendimiento visual y el rendimiento deportivo están íntimamente relacionados. Durante los juegos de invierno 1992, en Albertville, un sondeo hecho con la colaboración de 574 atletas reveló que el 34\% de ellos usaba anteojos, y que el 19\% de ellos usaba lentes de córnea (lentes quirúrgicamente colocados dentro del globo ocular).
Durante la competición, la adquisición de información es numerosa, móvil, a diversas distancias, en visión estática y dinámica, en visión central y periférica, proveniente de las tres dimensiones en el espacio, a veces con niveles muy bajos de luz. También es sabido que el equilibrio del atleta involucra el percibir y procesar adecuadamente información visual, vestibular y propioceptiva, por lo que los campeones necesitan de todos los componentes de su percepción visual (Corbe, 1994).

Las destrezas visuales que a saber influyen más en el deporte son: Agudeza visual, (especialmente la dinámica o en movimiento) visión periférica, percepción de profundidad o estereopsis, agudeza o exactitud de triangulación, velocidad y exactitud del movimiento del ojo, todas ellas mejorables por entrenamiento visual optométrico para ciertos autores como Sherman (1980). 
El examinar la sensibilidad visual del deportista, especialmente el de alto rendimiento, se considera una herramienta importante para determinar deficiencias tratables, e incluso potenciales inaptitudes (Corbe, 1994).

Situándonos en una variable visual específica, la visión periférica es de gran importancia motriz, al contribuir con el equilibrio locomotor dinámico así como control postural desde los tres años de edad y hasta la etapa adulta (Assaiante y Amblart, 1992). Además hay estudios sumamente interesantes que han encontrado que la visión periférica es susceptible de disminuir de forma aguda por estrés psicológico, exponiendo al deportista a una alta incidencia de lesiones físicas (Williams, Tonymon y Andersen,1991). También hay evidencia de influencia de tipo agudo del ejercicio sobre la visión periférica, la cual mejoró luego de realizar esfuerzo anaeróbico aláctico, anaeróbico láctico, así como aeróbico máximo y submáximo (Fleury y Bard, 1990). De igual forma, al hacer ejercicio mejoró de forma aguda la visión periférica en niños con distrofia muscular (Eickelbert, Kaylor, Less, Barunch y Megarr, 1983), así como los que presentaron espina bífida manifiesta (Krebs, Eickelbert, Krobath y Barunch, 1989). Por último, los jugadores de tenis de alto rendimiento resultaron tener mejor visión periférica y agudeza visual que los de nivel intermedio $y$ jugadores principiantes (Blundel, 1983).

Existe debate acerca de la entrenabilidad de ciertos componentes de la función visual; para la visión periférica, Jones (1997) encontró mejoría en las pruebas en tal variable tanto en los sujetos del grupo experimental (tratamiento para mejorar visión periférica) como en los del grupo control, (no tratamiento) por lo que atribuyó los cambios a fenómenos de aprendizaje en los test, más que a los tratamientos del grupo experimental. (por lo tanto en ese estudio la variable dependiente no resultó mejorada con el entrenamiento)

El efecto positivo agudo sobre la visión periférica en variadas poblaciones al realizar ejercicio físico probablemente se debe a un fenómeno de midriasis (dilatación de la pupila), debido al tono simpático aumentado por el ejercicio, fenómeno que a su vez disminuye la agudeza visual (Gannong, 1994).

En la variable de agudeza visual, la evidencia de efectos agudos inducidos por la actividad física no es escasa, aunque un poco inconsistente. Por un lado, la variable disminuyó luego de realizar 15 minutos de pedaleo en el cicloergómetro, en un ambiente de visión mesópica (nivel intermedio de luz), notándose más la disminución conforme se incrementó la carga de trabajo en el estudio de Ishigaki, Miyao, Ishihara, Sakakibara, Yamada, Furuta y Sacata (1991). Esto es compatible con la teoría de la midriasis y con parte del estudio de Watanable (1983), quién reportó que en 15 minutos de ejercicio e independientemente de la carga, la agudeza visual estática disminuyó, y que la misma aumentó apreciablemente a los nueve minutos siguientes a la finalización del esfuerzo. Sin embargo en este mismo estudio se examinó la agudeza visual kinética (en movimiento) la cual demanda mayor actividad cortical y que según el autor no varió al ejercitarse con una carga baja, aumentó con una carga moderada y disminuyó con la carga severa. El hecho que a una carga moderada la agudeza visual aumente es inconsistente con los estudios anteriormente mencionados $y$ con el fenómeno de midriasis, por lo que sería interesante insistir en esta modalidad de la agudeza visual (en movimiento) ya que el estudio de Watanable es de 1983 y es prácticamente el único que examina de ésta forma dicha variable. En consenso con Watanable, el estudio de Vlanov, (1980) también reporta efectos agudos positivos del ejercicio en la agudeza visual, en este caso estática, por lo que en resumen parte de la bibliografía señala que el efecto agudo es negativo ( la más reciente) mientras que otra parte indica que el efecto es positivo (la más antigua)

Existen reportes de menor resistencia cardiovascular, flexibilidad y balance en individuos con deficiencias visuales; éste efecto se produce por la falta de información sensorial de los individuos de mayor 
problemática visual a la hora de ejecutar las respectivas pruebas (Skaags y Jopper, 1996).

Finalmente, Spurgeon, French, Rivers, Bailey y Ellisord (1989), no encontraron relación entre la función visual y el promedio de bateo en béisbol, pero si encontraron relación significativa con el porcentaje de fildeo.

Debido a la evidencia existente de que la actividad física ejerce influencias sobre las funciones visuales, este estudio pretendió examinar la existencia de un posible efecto agudo negativo de la deshidratación física (no ocular) inducida por ejercicio sobre la agudeza visual estática.

Es sabido que la deshidratación tiene un efecto progresivamente negativo sobre el rendimiento en ejercicio aún a niveles tan bajos como el 1\% (ACSM 1996, Ekblom, Greenleaf, y Hermansen, 1970) el 2\% (Armstrong, 1985) y el 3\% (Sawka, 1992). La hipohidratación en combinación con el calor perjudica muchas variables fisiológicas durante el ejercicio, ocasionando un menor volumen sanguíneo que incide en el gasto de eyección y llenado ventricular, que no pueden compensarse con el consecuente aumento de la frecuencia cardiaca (Coyle 1998), lo que disminuye el rendimiento físico al incapacitar al sistema cardiovascular para mantener el gasto cardiaco y que a la vez disminuye la perfusión tisular (González, Mora, Rodríguez, Below y Coyle, 1997). La deshidratación y el calor ambiental inducen a una mayor temperatura corporal capaz de llevar a la fatiga de forma prematura, pues el calor corporal influye de manera negativa sobre el funcionamiento del sistema nervioso central y la función mental, afectando los centros motores, la capacidad de reclutamiento de fibras nerviosas y musculares, e inclusive disminuyendo la motivación hacia el rendimiento físico (Nielsen, Savard, Richter, Hargreaves y Saltin 1990).

La base experimental para atribuir la fatiga física prematura a la inhibición de las funciones cerebrales por el calor corporal se deriva del hecho de que los sujetos que se ejercitan en el calor se fatigan más rápidamente que en otras condiciones ambientales, a pesar de encontrar importantes remanentes de glucógeno muscular en ellos a la hora de no poder continuar con el ejercicio, así como no presentar perturbaciones metabólicas considerables (Febbraio, Parking, Baldwing, Zhao y Carey, 1995). En el caso extremo de un golpe de calor, se da disfunción severa del sistema nervioso central, que se manifiesta por pérdida de la coordinación motora, delirio, inconciencia y coma.

Uno de los principales beneficios de la rehidratación en el ejercicio, es prevenir la elevación adicional de la temperatura corporal, que se da aún en los niveles más bajos de deshidratación. La ingesta de fluidos mejora la perfusión tisular, al mantener el volumen sanguíneo al tiempo que promueve un mayor flujo sanguíneo epitelial, produciéndose las mayores tasas de flujo sanguíneo cuando se ingirieron los mayores volúmenes de líquido durante la actividad física (Montain y Coyle, 1992).

Es sabido que la corteza cerebral aumenta su flujo sanguíneo en las zonas encargadas de realizar la actividad que se desempeña; así por ejemplo, el movimiento de una mano provoca un aumento del flujo sanguíneo sólo en la zona sensitivo-motora contralateral y en la corteza premotora (Berne y Levy, 1998).

Hablar, leer, y otros estímulos a los centros cerebrales llevan aparejado un aumento de flujo sanguíneo en las zonas responsables de la corteza; tal fenómeno se da también cuando demandamos gran atención visual y concentración, como al leer, en donde se ve un aumento de la irrigación sanguínea parietal y occipital de la corteza cerebral contralateral (Berne y Levy 1998). Por otro lado, debemos recordar que entre los diferentes tejidos corporales, el cerebro es el que menos tolera la isquemia. La interrupción de flujo sanguíneo cerebral durante 5 segundos, provoca la pérdida de la conciencia (Berne y Levy 1998), ¿podría la redistribución del flujo sanguíneo inducida por el ejercicio o los cambios en las características del plasma debido a la deshidratación afectar funciones nerviosas superiores al menos levemente? 
La deshidratación podría según la evidencia, afectar la función visual general, al generar un flujo sanguíneo inadecuado por su bajo volumen y alta osmolalidad, así como al elevar la temperatura corporal central afectando las funciones del sistema nervioso central y periférico.

Se esperaba encontrar un efecto agudo negativo de la deshidratación inducida por ejercicio, sobre la agudeza visual, componente íntimamente relacionado con el rendimiento físico, con creciente importancia conforme mayor es el nivel atlético, donde las mínimas diferencias deciden quién es el mejor.

\section{METODOLOGÍA}

\section{Participantes}

Participaron en su totalidad 15 mujeres de edades desde los 18 hasta los 40 años. Todas las participantes tenían un nivel de acondicionamiento físico tal, que les permitía soportar las demandas del estudio, así como poseer umbrales y tasas de sudoración que facilitaran su deshidratación.

Se evitó que las mujeres estuvieran en los días próximos a su ciclo menstrual, (10 días) para evitar variaciones temporales en el líquido corporal.

\section{Instrumentos de medición}

La agudeza visual se determinó con la cart de Snellen de 11 niveles (Del Río, 1988), para incrementar su sensibilidad. (en muchos casos se usan de únicamente 8 niveles de puntuación) (Figura 1). Se desistió de utilizar pruebas en computadora debido a que se corría el riesgo de afectar los resultados por cambios en el brillo, el contraste y en los colores que el uso de monitores puede generar, y que de darse entre una condición y otra representarían estímulos diferentes que no podrían compararse para realizar inferencias.

El peso corporal se determinó con una romana electrónica de $0.005 \mathrm{Kg}$ de sensibilidad (determina el peso en rangos de 5 gramos).
La frecuencia cardiaca se determinó con monitores electrónicos de ritmo cardiaco marca Polar ${ }^{\circledR}$.

Figura 1. Carta de Snellen.

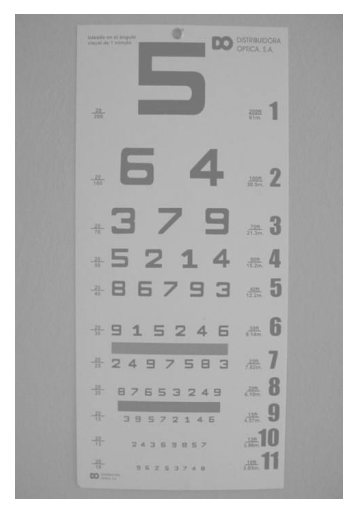

\section{Diseño experimental}

Se empleó un diseño de medidas repetidas, considerando las condiciones control y experimental (rehidratación y deshidratación respectivamente).

Cada sujeto realizó ambas condiciones con pre y post test en cada una de ellas, en cuanto a su agudeza visual.

\section{Procedimientos de medición}

Con el fin de asegurar la euhidratación al iniciar las condiciones, el día anterior a cada una las participantes bebieron de 1.5 a 2 litros de agua, y entre 250 y $500 \mathrm{ml}$ de agua unas 2 horas antes de ejercitarse; se les restringió el consumo de alimentos o productos que pudieran deshidratarlas (bebidas gaseosas, cafeína, alcohol y alimentos ricos en sodio) desde 24 horas antes de las pruebas y orinaron antes del inicio de cada condición, controlando así los niveles de hidratación. Para determinar si no consumieron alimentos restringidos por el protocolo del estudio, así como si tomaron suficiente líquido, se les efectuó un registro de alimentación de las últimas 24 horas el cual se efectuó por escrito.

En la condición de deshidratación (experimental) los sujetos no repusieron la pérdida de fluidos de forma total, pero se les 
administró ciertas dosis de líquido (agua. Generalmente $250 \mathrm{ml}$ ) para evitar cualquier efecto de nocebo a la hora de realizar las pruebas visuales. Se pretendió inducir un nivel de deshidratación de un $2 \%$ de su peso corporal. Ningún sujeto dio muestras de malestar producto de las condiciones o de alguna otra eventualidad.

En la condición control (rehidratación) se procedió igual que en la experimental, pero con una diferencia, pues los sujetos bebieron entre 250 y $600 \mathrm{ml} \mathrm{de}$ agua (según su peso y sudoración) cada 15 minutos, o una cantidad tan similar como les fue posible sin sentirse incómodas, (pues algunas no estaban familiarizadas con protocolos de rehidratación) para reponer lo mejor posible las pérdidas de líquido .

Las condiciones se llevaron a cabo en bicicletas estacionarias, (cicloergómetro Monark) a intensidades que oscilaron entre el $65 \%$ y el $85 \%$ de su frecuencia cardiaca máxima calculada por la fórmula de Karvonen (ACSM, 1998) y controlada con los monitores de ritmo cardiaco. Las mismas se realizaron en el laboratorio de clima controlado de la Escuela de Educación Física y Deportes de la Universidad de Costa Rica, con temperaturas de 28 a 32 grados centígrados y niveles de humedad de $80 \%$ a $100 \%$.

Antes del inicio de cada condición, se midió la agudeza visual haciendo uso de los diagramas de Snellen, los cuales ofrecen una valoración de la variable en términos de pies. La prueba se realizó para ambos ojos simultáneamente así como para cada uno de forma individual, y se utilizó la misma cantidad de luz artificial en cada prueba. Cuando se examinó un solo ojo a la vez, se mantuvo abierto el otro ojo, para evitar distorsiones en el ojo examinado producto del reflejo fotomotor consensual (Willis y Williams en Berne, 1998). Inmediatamente se procedió a pesar a los sujetos con el fin de controlar el proceso de rehidratación o de deshidratación. La prueba en el diagrama de Snellen se repitió después de aplicar la condición correspondiente con el fin de determinar los cambios en la visión producto de cada una de ellas, no sin antes dejar pasar un lapso corto de tiempo (15 min) en el que los sujetos se terminaron de secar, se pesaron y se recuperaron del esfuerzo físico; tiempo que a la vez permitió la normalización de los efectos de midriasis inducidos por la activación simpática que genera la actividad física y que como es sabido, y citado anteriormente, afecta negativamente la agudeza visual, hasta 10 minutos después de finalizado el ejercicio (Watanable 1983). De esta forma se controló cualquier posible confusión en los datos producto de la actividad física en sí misma.

El grado de deshidratación se determinó considerando el peso corporal antes de iniciar la condición y después de ella, y se hizo relativa la pérdida de peso al porcentaje de masa corporal correspondiente. Los sujetos se pesaron siempre sin ropa y con su cuerpo seco y realizaron dos intervalos de treinta minutos de actividad física, con un descanso de 15 minutos entre cada una de ellas en el que eran pesados para monitorear la progresión de la condición. Cuando fue necesario se procedió a realizar un intervalo adicional de 15 minutos de duración, lo cual sucedió en pocos casos.

Luego de aplicar cada una de las condiciones y de realizar las pruebas respectivas, se veló por una adecuada reposición de fluidos, bebiendo incluso más cantidad del líquido que creyeron los sujetos era necesario (aprox. $250 \mathrm{ml} / \mathrm{kg}$ de peso perdido).

La aplicación de las condiciones se llevó a cabo de forma aleatoria para cada sujeto, y se dejó pasar en casi todos los casos una semana de tiempo entre las mismas. En algunos sujetos, y a solicitud de ellos, este periodo se amplió, siempre y cuando no interfiriera con la necesidad de que faltaran al menos 10 días para el inicio del ciclo menstrual.

En cuatro de los sujetos se monitoreó la temperatura corporal central antes y después de las condiciones, con el fin de determinar cuanto aumentaba en cada condición, información indispensable para la discusión. No se realizó esta medición en la totalidad de los sujetos porque la mayoría estuvo en desacuerdo para firmar el 
consentimiento, por lo que se realizó de manera voluntaria en la mayor cantidad posible personas en la muestra.

\section{Análisis estadístico}

El análisis estadístico se realizó utilizando tres anovas de dos vías (medidas repetidas) para la variable dependiente, (agudeza visual) en cada una de las condiciones descritas, una para los dos ojos medidos a la vez así como una para cada ojo medido por separado.

También se realizó una prueba $t$ student para determinar si existieron diferencias significativas entre los pesos iniciales en cada condición, que de darse al trabajar con mujeres implicaría una posible retención de líquido producto de los fenómenos normales del ciclo menstrual.

\section{RESULTADOS}

El cuadro 1 muestra las características de los sujetos, tales como edad y peso así como los niveles de deshidratación y de rehidratación a que fueron inducidos.

El peso inicial en la condición de deshidratación no presenta diferencias significativas con respecto al peso inicial de la condición rehidratación, como lo indica la $t$ student realizada con ambos promedios $(\mathrm{p}=$ 0.173). Esto demuestra estadísticamente que entre ambas condiciones no se dieron alteraciones significativas en el peso inicial, gracias a la euhidratación interpuesta y el poco tiempo transcurrido entre mediciones (1 semana) lo que permitió controlar la presencia de alteraciones importantes del líquido corporal producto del ciclo menstrual.

La variación de peso en la condición de rehidratación corresponde al aumento o disminución del peso corporal inicial en la condición de rehidratación debido al volumen de agua consumido determinado por el protocolo de reposición de fluidos.

Los resultados obtenidos al examinar la agudeza visual son expresados en una escala del 1 al 11, siendo 1 correspondiente con una visión de 20/50 (visión sumamente pobre) 8 para una visión de 20/20 (visión normal) y 11 para una agudeza visual de 20/10 (el doble de agudeza visual normal).

Cuadro 1. Descripción de los sujetos antes y después de la aplicación de las condiciones.

\begin{tabular}{lcc}
\hline \multicolumn{1}{c}{ Característica } & Promedio & $\mathbf{\pm}$ D.E. \\
\hline Edad (Años) & 24.72 & 4.24 \\
P. I. D. (kg) & 54.24 & 6.04 \\
P. I. R. (kg) & 54.07 & 6.33 \\
G. D. (\%) & 2.01 & 0.21 \\
G. R. (\%) & 2.17 & 0.24 \\
V.P. R. (\%) & 0.12 & 0.15 \\
\hline
\end{tabular}

$\mathrm{PID}=$ peso inicial el día de deshidratación

PIR= peso inicial el día de rehidratación

GD= grado de deshidratación inducido

GR= grado de rehidratación inducido*

VPR= variación del peso inducido por la rehidratación. Aumento del peso.

*equivale al porcentaje del peso corporal que los sujetos bebieron durante la rehidratación.

El cuadro 2 muestra los promedios de agudeza visual en cada condición (pre y post test) tanto para ambos ojos mirando simultáneamente como para cada uno de ellos por separado.

Cuadro 2. Valores de la agudeza visual antes y después de las condiciones.

\begin{tabular}{lcc}
\hline Condición & Pre test & Post test \\
\cline { 2 - 3 } & Prom \pm DS & Prom \pm DS \\
\hline Desh 2 ojos & $7.26 \pm 1.33$ & $7.26 \pm 1.16$ \\
Desh ojo D & $6.93 \pm 1.48$ & $7.00 \pm 1.25$ \\
Desh ojo I & $7.13 \pm 1.30$ & $7.07 \pm 1.33$ \\
Reh 2 ojos & $7.93 \pm 1.16$ & $7.80 \pm 1.26$ \\
Reh ojo D & $7.40 \pm 1.18$ & $7.53 \pm 1.35$ \\
Reh ojo I & $7.47 \pm 1.19$ & $7.67 \pm 1.18$ \\
\hline
\end{tabular}

El análisis de varianza efectuado para ambos ojos mirando simultáneamente indica que no existieron diferencias significativas entre la agudeza visual inicial y final en ninguna de las condiciones, lo que indica que estas (deshidratación y rehidratación) no afectaron positiva o negativamente la agudeza visual $(p=0.433)$. Se encontraron diferencias significativas en la agudeza visual entre ambas condiciones, a favor de la rehidratación, lo que indica que algunos días los mismos sujetos presentaban diferencias en su agudeza visual independientemente de la condición interpuesta $(\mathrm{p}=0.012)$. Por último, 
no se encontró interacción significativa entre condiciones y mediciones de pre test y pos test, lo que indica que los tratamientos no efectuaron efectos distintos sobre la variable dependiente $(\mathrm{p}=0.433)$.

Con respecto al ojo derecho mirando por sí solo, los efectos observados son los mismos, pues no se encuentran diferencias significativas entre los resultados de pre y post test en ninguna de las condiciones $(\mathrm{p}=$ 0.510) se hallaron diferencias significativas para la agudeza visual total entre las condiciones a favor de la rehidratación ( $\mathrm{p}=$ 0.030) y no existió interacción significativa entre condiciones y mediciones, por lo que los tratamientos no afectaron diferenciadamente a la agudeza visual $(\mathrm{p}=$ 0.774). Esto último es de esperarse, pues ninguna de las condiciones causó algún efecto sobre la agudeza visual, por lo que una posible interacción queda imposibilitada.

En el caso del ojo izquierdo mirando por sí solo, el patrón cambió, pues aunque no existieron diferencias significativas entre los resultados de la medición de agudeza visual de pre test y post test en ambas condiciones, ( $\mathrm{p}=0.334$ ) (lo cual coincide con los dos análisis anteriores) ni se dio por lo tanto una interacción significativa entre condiciones y mediciones de pre y post test $(p=0.164)$, tampoco se dio (y esto solo en el ojo izquierdo) diferencia significativa entre la agudeza visual de una condición con respecto a la otra, lo que indica que esta se mantuvo constante entre sesiones, con un lapso promedio de 7 días de diferencia entre cada medición $(\mathrm{p}=0.079)$.

Los gráficos 1, 2 y 3 ilustran los comportamientos de la agudeza visual en las condiciones, siendo medidos ambos ojos, sólo ojo derecho y sólo ojo izquierdo respectivamente.

En los gráficos 1 y 2 las diferencias se dan sólo entre el promedio de las condiciones y nunca entre el pre y post test de las mismas. En el gráfico 3, no existen diferencias significativas en ningún tipo de comparación.
Gráfico 1. comportamiento de la agudeza visual en ambos ojos durante la aplicación de las condiciones.

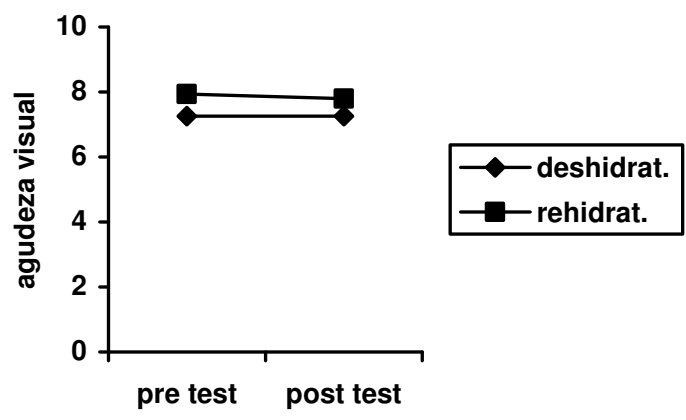

Gráfico 2. comportamiento de la agudeza visual del ojo derecho examinado por sí solo durante la aplicación de las condiciones.

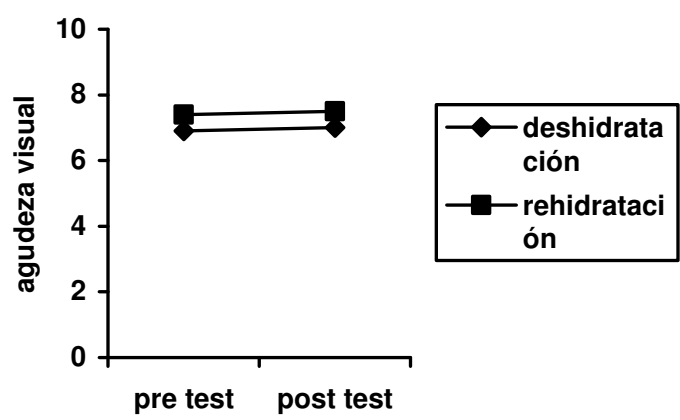

Gráfico 3. comportamiento de la agudeza visual del ojo izquierdo examinado por sí solo durante la aplicación de las condiciones.

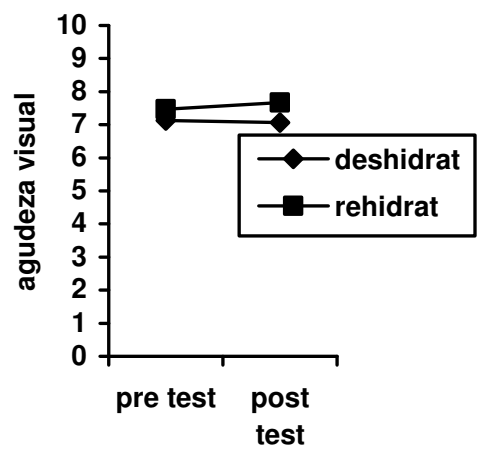

Finalmente, en el cuadro 3 se ilustra el comportamiento de la temperatura rectal de los cuatro sujetos medidos en ambas condiciones. Se aprecia la tendencia esperada, que implica un incremento de temperatura mayor en deshidratación que en rehidratación. 
Cuadro 3. Temperaturas rectales $\left({ }^{\circ} \mathrm{C}\right)$ de los participantes en las dos condiciones experimentales.

\begin{tabular}{ccccc}
\hline Sujeto & \multicolumn{2}{c}{ Deshidratación } & \multicolumn{2}{c}{ Rehidratación } \\
\hline & Inicial & Final & Inicial & Final \\
\hline 1 & 37.3 & 38.3 & 37.2 & 37.5 \\
2 & 37.1 & 38.1 & 37.0 & 37.3 \\
3 & 37.3 & 38.1 & 37.1 & 37.4 \\
4 & 37.2 & 38.2 & 37.0 & 37.5 \\
\hline
\end{tabular}

\section{DISCUSIÓN}

Las pérdidas de fluidos por deshidratación se distribuyen en varias proporciones entre el plasma, (10 a $11 \%$ del líquido perdido) el liquido intracelular (aprox. $50 \%$ del líquido perdido) y el extracelular (el 40\% restante), según Costill (1977). La disminución en el volumen plasmático que acompaña a la deshidratación puede ser de particular importancia al influenciar negativamente variables claves para el rendimiento, como la capacidad de trabajo físico. El flujo sanguíneo a los músculos debe ser mantenido a altos niveles para suministrar el oxígeno y los substratos, además de ser paralelamente necesario un alto flujo sanguíneo a nivel cutáneo para perder calor por convección con el medio ambiente (Nadel 1980).

En casos de temperaturas ambientales elevadas (como en el presente estudio) y cuando el volumen sanguíneo ha sido disminuido por pérdidas en sudoración por ejercicio, es difícil mantener los requerimientos de flujo sanguíneo en ambas zonas (músculos y piel).

Ante tal situación es el flujo sanguíneo cutáneo el que se ve disminuido, permitiendo mantener la presión venosa central y la perfusión muscular, con el precio de reducir las pérdidas de calor y permitir la elevación de la temperatura central (Rowel, 1997).

En los sujetos de este estudio cuya temperatura rectal fue determinada antes y después de las condiciones, se reflejaron claramente estas tendencias, pues la temperatura central aumentó notoriamente en la condición deshidratación y no así en la rehidratación, donde los aumentos fueron menores. Dichos valores se obtuvieron para confirmar el apego del estudio a la teoría, y para tener certeza de que las condiciones no sólo se llevaron a cabo, también ejercieron los efectos esperados. No se realizaron análisis estadísticos con los datos de temperatura rectal.

Se ha reportado en numerosas investigaciones que niveles de deshidratación del 1\% (ACSM, 1996), ó 2\% (Armstrong, 1985), de la masa corporal total afectan negativamente el rendimiento físico. Tal efecto se da tanto en eventos de alta como de baja intensidad. Algunos estudios demostraron que la deshidratación a un 2,5\% de la masa corporal disminuyó en un $45 \%$ la capacidad de realizar ejercicio de alta intensidad (Nielsen, Kubica, Bonnesen, Rassmusen, Stocklosa y Wilk, 1981) y además pérdidas del $1,8 \%$ afectan negativamente la tolerancia al ejercicio (Walsh, Noakes, Hawley y Dennis, 1994). Se ha incluso mencionado que pérdidas de fluidos aún menores pueden afectar el rendimiento competitivo en deportes en que las diferencias entre el ganador y perdedor son extraordinariamente pequeñas, tales que los métodos de laboratorio talvez no sean lo suficientemente sensibles para detectar tan pequeñas variaciones en el rendimiento (Maughan y Murray 2000).

El hecho de que el sudor sea hipotónico con respecto a los fluidos corporales, genera que la deshidratación produzca un incremento en la osmolalidad plasmática que a su vez puede afectar la capacidad termoregulatoria del organismo. Ya ha sido demostrada una relación lineal existente entre la osmolalidad plasmática y la temperatura corporal durante la actividad física (Greenlaf y Castle, 1974, Harrison, Edwards, y Fennessey, 1978). Esta relación causa-efecto contribuye con el aumento de la temperatura corporal (además de la producción incrementada de calor) en eventos mayores de 30 minutos de duración (Fortney, Vroman, Beckett, Permutt y LaFrance, 1988).

$\mathrm{Si}$ la actividad física genera deshidratación que es capaz de afectar la capacidad termorregulatoria, también genera 
pérdidas de líquido vascular que no es detectado por el método de pesaje y que disminuye la perfusión tisular. En ejercicios de alta intensidad, de corta duración, el volumen plasmático disminuye por una redistribución interna del líquido que lo traslada de los vasos al tejido muscular adyacente (el conocido edema tisular agudo que se genera por aumentos de la presión hidrostática vascular). También en los músculos activos se da glucogenólisis y un consecuente aumento de sus productos paralelos: El lactato y el piruvato incrementan su concentración, así como los intermediarios fosforilados de la glucólisis. Este incremento en la osmolalidad de las células aumenta la presión oncótica sobre el líquido intersticial y plasmático. Ambos fenómenos producirán disminuciones en el líquido vascular aún en ausencia de cambios en el peso corporal (Maughan y Murray 2000).

En actividades de muy alta intensidad se dan disminuciones en el líquido vascular de hasta un $12 \%$ con respecto al nivel preejercicio. Tomando en cuenta que el volumen plasmático es de 41 a $44 \mathrm{ml} / \mathrm{kg}$ de peso, esto representa disminuciones de $300 \mathrm{a} 400 \mathrm{ml}$ en una persona de $70 \mathrm{~kg}$. (Lentner, 1984). Esta disminución plasmática presenta niveles de variabilidad entre sujetos, y existen reportes de disminuciones del $20 \%$ del volumen plasmático en ejercicios con intensidades cercanas al 100\% (Maughan y Murray 2000).

En casos de ejercicio prolongado, (como en el presente estudio) se da una disminución inicial del volumen plasmático por la incorporación de agua a los músculos activos. Este fenómeno sin embargo, involuciona alcanzándose niveles cercanos (no iguales) a los originales luego de una hora de ejercicio (duración promedio aplicada en este estudio). La Figura 2 ilustra este fenómeno.

Es sabido que esta reposición del líquido vascular conforme transcurre el tiempo se da con más claridad en el trote que en el ciclismo (Maughan y Murray 2000), por lo que los sujetos aquí estudiados posiblemente fueron afectados por este fenómeno, auque esto no fue determinado ya que el peso corporal (sus cambios) no es suficiente para detectar tales fluctuaciones (no se detectan cambios entre compartimentos de líquido corporal).

Fig. 2. Cambios en el volumen plasmático durante ejercicio de ciclismo prolongado al 70\% de intensidad, con los sujetos siendo deshidratados, hidratados con agua, o con solución de CHO y electrolitos en intervalos durante el ejercicio.

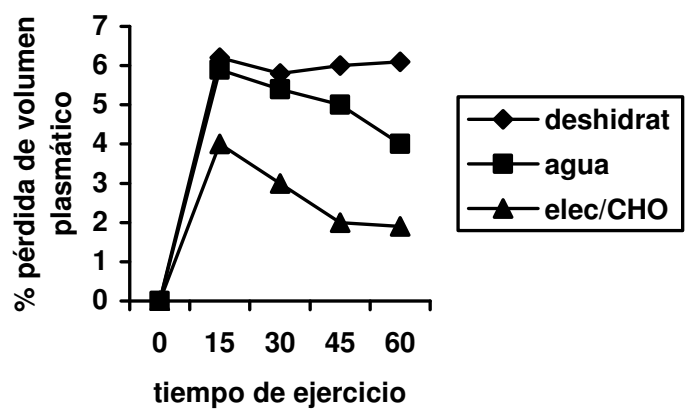

Datos de Maughan y otros (1996). (Nótese la pérdida final de líquido plasmático a pesar de la rehidratación con agua, protocolo utilizado en este estudio)

Los sujetos estudiados se comportaron conforme a lo esperado en cada una de las condiciones. En el caso de la deshidratación perdieron líquido corporal en un $2.01 \%$ en promedio, aumentando su temperatura corporal, y estando expuestos a los fenómenos fisiológicos descritos anteriormente. En la condición de rehidratación el peso corporal presentó cambios mucho menores $(+0.12 \%)$ y la temperatura corporal central aumentó menos que en la deshidratación.

En este caso a pesar del buen desempeño en la aplicación de las condiciones, no fue posible detectar cambios significativos en la agudeza visual a través del uso de los diagramas de Snellen, pues las diferencias en la variable estudiada de pre a post test en cada condición no fueron significativas. Solamente fueron significativas las variaciones en la agudeza visual de un día a otro (con una semana de diferencia) independientemente de la condición interpuesta, lo que se da en muchas personas normalmente y sin guardar relación 
con la práctica de actividad física, pues la agudeza visual presenta fluctuaciones agudas pasajeras en función de factores de estímulo (iluminación, brillo, contraste, fondo $\mathrm{y}$ tiempo de exposición, todos mantenidos constantes), y factores retinianos como lo son la fatiga de los conos, siendo esta última la que explicaría las variaciones encontradas en la agudeza visual. Los cambios encontrados, por lo tanto, según la teoría así como el análisis estadístico y la forma en que se dieron, se deben a factores externos en su totalidad y no a efectos de alguna de las condiciones aplicadas.

El aumento de la temperatura, la pérdida y redistribución del líquido corporal así como el incremento de la osmolalidad plasmática no fueron suficientes en este caso para disminuir la agudeza visual sensiblemente. Se podría evaluar la variable dependiente haciendo uso de instrumentos aún más sensibles que los diagramas de Snellen, pero los eventuales hallazgos (si se dan) perderían validez externa conforme más pequeños sean los cambios detectados, por lo que su influencia en el campo sería cuestionable. El realizar estos estudios con los mismos y otros métodos de medición en condiciones de deshidratación mayores o en otros tipos de estrés producidos por el ejercicio son los pasos a seguir que análisis como este hacen llamado, con el objeto de dilucidar los fenómeno fisiológicos que ocurren durante la practica de la actividad física.

\section{REFERENCIAS}

American College of Sport Medicine. (1996). ACSM Position Stand on Exercise and Fluid Replacement. Med. Sci. Sports Exerc, 28(1), ivii.

Armstrong, L. E., Costill D. L., y Flink, W. J. (1985). Influence of diuretic-induced dehydration on competitive running performance. Med. Sci. Sports Exerc, 17, 456-461.

Assaiante, C., y Amblard, A. (1992) Peripheral Vision and Age-Related Differences in Dynamic Balance. Human Movement Science, 11(5), 533548.

Berne, R., y Levy, M. (1998). Fisiología. Sistema Cardiovascular (2da Ed.). Madrid: Headcourt Brace.
Blundel, N. L. (1983). Critical Visual-Perceptual Attributes of Championship Level Tennis Players. En Howel, M. L., y Wilson, B. D. (Eds.). Kinesiological Sciences. Pp. 51-59.

Corbe, C. (1994). Exploration of Functional Vision of The Sportperson. Science and Sports, 9(1), 1-10.

Costill, D. L. (1977). Sweating: its composition and effects on body fluids. Ann. N. Y. Acad. Sci., 301, 160-174.

Coyle, E. F. (1998). Cardiovascular Drift During Prolonged Exercise and the Effects of Dehydration. Int. J. of Sport. Med., 19, S121S124.

Eckblom, B., Greenleaf C. J., Greenleaf, J. E., y Hermansen, L. (1970). Temperature Regulation During Exercise Dehydration in man. Acta Physiol. Scand., 79, 475-483.

Eickelberg, W., Kaylor, P., Less, M., Barunch, I., y Megarr, J. (1983) Effects of Passive Physical Exercise on Peripheral Vision in Muscular Dystrophic Children. Perceptual and Motor Skills, 56(1), 167-170.

Febbraio, M. A., Parking, J. A., Baldwin, L., Zhao, S., y Carey, M. F. (1995). Metabolic Indices of Fatigue in Prolonged Exercise at Different Ambient Temperatures. Abstracts of Poster Presentations: Dehydration, Rehydration And Exercise in the Heat. Nottingham, England.

Fleury, M., y Bard, C. (1990). Metabolic Fatigue and Performance of Visual Tasks. Canadian Journal of Sport Sciences, 15(1), 43-50.

Fortney, S. M., Vroman, N. B., Beckett, W. S., Permutt, S., y LaFrance, N. D. (1988). Effect of exercise hemoconcentration and hyperosmolality on exercise responses. Journal of Applied Physiology, 65, 519-524.

Gannong, W. (1994). Fisiología Médica (14 ${ }^{\mathrm{a}}$ Edición). México, D.F.: Manual Moderno, S. A..

Del Río, G. (1988). Fisiología Óptica. ( $3^{\text {ra }}$ Edición). México, D. F.: Manual Moderno, S. A..

Gonzalez-Alonso, J., Mora Rodríguez, R., Below, P. R., y Coyle, E. F. (1997). Dehydration Madkerly impairs Cardiovascular Function in Hyperthermic Endurance Athletes During Exercise. Journal of Applied Physiology, 82(4), 1229-1236.

Greenleaf, J. E., Castle, B. L., y Card, D. H. (1974). Blood electrolytes and temperature regulation during exercise in man. Acta Physiologica Polonia, 25, 397-410.

Harrison, M., H., Edwards, R. J., y Fennessey, P. A. (1978). Intravascular Volume and tonicity as factors in the regulation of body temperature. Journal of Applied Physiology. 44, 69-75.

Ishigaky, H., Miyao, M., Ishihara S.Y., Sakakibara, H., Yamada, S. H., Furuta, M., y Sakata, T. (1991). The Deterioration of Visual Acuity by Exercise Under a Mesopic Vision Environment. Journal Of Sport Medicine and Physical Fitness, 31(2), 272-276.

Jones, R. L. (1997). Improving Peripheral Vision in Athletes. Journal of the International Council for 
Health, Physical Education, Recreation, Sport and Dance, 33(3), 55-58.

Krebs, P., Eickelbert, W., Krobath, H., y Baruch, I. (1989). Effects of Physical Exercise on Peripheral Vision and Learning in Children With Spina Bifida Manifesta. Perceptual and Motor Skills, 68(1), 167-174.

Lee, P. P., Spritzer, K., y Hays, R. D. (2000) The impact of blurred vision on functioning and wellbeing. British Journal of Ophthalmology, 84, 165-170.

Lentner, C. (1984). Geigy Scientific tables. Vol. Physical Chemistry, Composition of blood, Hematology, somatometric data. ( $8^{\text {th }}$ ed.). CibaGeigy, Basle. Pag. 65.

Maughan, R. J., Bethell, L. R., y Leiper, J. B. (1996). Effects of ingested fluids on exercise capacity and on cardiovascular and metabolic responses to prolonged exercise in man. Experimental Physiology, 81, 847-859.

Maughan, R. J., y Murray, R. (2000). Sport Drinks. basic science and practical aspects. Boca Raton, FL: CRC Press.

Montain, S. J., y Coyle, E. F. (1992). The Influence of Graded Dehydration on Hyperthermia and Cardiovascular Drift During Exercise. Journal of Applied Physiology, 73, 1340-1350.

Nadel, E. R. (1980). Circulatory and thermal regulations during exercise. Fed. Proc. 39, 1491-1497.

Nielsen, B., Kubica, R., Bonnesen, A., Rasmussen, I. B., Stoklosa, J., y Wilk, B. (1981). Physical work capacity after dehydration and hypertermia. Scand. Journal Sport Sciences, 3,2-10.

Nielsen, B., Savard, G., Richter, E.A., Hargreaves, M., y Saltin, B. (1990). Muscle Blood Flow and Muscle Metabolism During Exercise and Head Stress. Journal of Applied Physiology, 69, 10401046.
Rowel, L. B. (1997). Human circulation. New York: Oxford University Press.

Sawka, M. N. (1992). Physiological Consequences of Dehydration: Exercise Performance and Thermoregulation. Med. Sci. Sport and Exerc, 24, 657-670.

Sherman, A. (1980). Overview of Research Information Regarding Vision and Sports. Journal of the American Optometric Association, 51(7), 661666.

Skaggs, S., y Hopperr, C. (1996). Individuals With Visual Impairments: A Review Of Psychomotor Behavior. Adapted Physical Activity Quarterly 13(1), 16-26.

Spurgeon, J. H., French, K. E., Rivers, J. B., Bailey, J. G., y Ellisord, D. B. (1989). The Relation Of Selected Eye Functions to Batting and Fielding Performance in Baseball. Journal of Applied Research in Coaching and Athletics, 4(4), 243254.

Vlanov, E. (1980). Visual Effects of Exercise: Sensory or decision Criterion Change. Perceptual and Motor Skills, 51(3, -pt- 1), 688-690.

Walsh, R. M., Noakes, T. D., Hawley, J. A., y Dennis, S. C. (1994). Impaired high intensity cycling performance time at low levels of dehydration. International Journal of Sports Medicine, 15, 392-398.

Watanable, Y. (1983). Effect of 15 Minute Bicycle Workload on Static and Kinetic Visual Acuities. Journal of Sport Medicine and Physical Fitness, 23(4), 373-381.

Williams, J. M., Tonymon, P., y Andersen, M. V. (1991). The Effects of Stressors and Coping Resources on Anxiety and Peripheral Narrowing. Journal of Applied Sport Psychology, 3(2), 126141. 